\title{
The performance of the MROI fast tip-tilt correction system
}

\author{
John Young ${ }^{a}$, David Buscher ${ }^{a}$, Martin Fisher ${ }^{a}$, Christopher Haniff $^{a}$, Alexander Rea ${ }^{a}$, Eugene \\ Seneta ${ }^{a}$, Xiaowei $\operatorname{Sun}^{a}$, Donald Wilson ${ }^{a}$, Allen Farris $^{b}$, and Andres Olivares ${ }^{b}$ \\ ${ }^{a}$ Cavendish Laboratory, University of Cambridge, JJ Thomson Avenue, Cambridge CB3 0HE, \\ UK; \\ ${ }^{b}$ Magdalena Ridge Observatory, New Mexico Institute of Mining and Technology, 801 Leroy \\ Place, Socorro, NM, 87801
}

\begin{abstract}
The fast tip-tilt (FTT) correction system for the Magdalena Ridge Observatory Interferometer (MROI) is being developed by the University of Cambridge. The design incorporates an EMCCD camera protected by a thermal enclosure, optical mounts with passive thermal compensation, and control software running under Xenomai real-time Linux. The complete FTT system is now undergoing laboratory testing prior to being installed on the first MROI unit telescope in the fall of 2014. We are following a twin-track approach to testing the closed-loop performance: tracking tip-tilt perturbations introduced by an actuated flat mirror in the laboratory, and undertaking end-to-end simulations that incorporate realistic higher-order atmospheric perturbations. We report test results that demonstrate (a) the high stability of the entire opto-mechanical system, realized with a completely passive design; and (b) the fast tip-tilt correction performance and limiting sensitivity. Our preliminary results in both areas are close to those needed to realise the ambitious stability and sensitivity goals of the MROI which aims to match the performance of current natural guide star adaptive optics systems.
\end{abstract}

Keywords: interferometry, tip-tilt correction

\section{INTRODUCTION}

The Magdalena Ridge Observatory Interferometer ${ }^{1}$ is being designed to deliver an interferometric sensitivity similar to that of current natural guide star adaptive optics, i.e. much better than many current interferometric arrays. One important aspect of its design philosophy has been to engineer each of its critical opto-mechanical subsystems such that none - in as far as this is possible - will fail well before the sensitivity limits of any of the others are reached. In this way we have hoped to ensure that expensive sub-systems are not compromised by inadequate performance of others, and have tried to balance the array's challenging technical goals across the interferometer as a whole.

In this paper we report on the laboratory testing of the as-built MROI Fast Tip-Tilt correction and Narrowfield Acquisition System (FTT/NAS). This system will sit on the Nasmyth optical table at each Unit Telescope (UT) in the array, and will pick off the shorter wavelength (initially with $\lambda<1 \mu \mathrm{m}$ ) light collected by the UT and use this for target acquisition and for high speed correction of atmospheric tip-tilt fluctuations of the light being sent for interferometric combination.

The FTT/NAS has been designed and fabricated at the Cavendish Laboratory of the University of Cambridge, and the first production unit is currently being tested in the laboratory in readiness for deployment in New Mexico in the fall of 2014. The preliminary design for the MROI FTT/NAS has been presented elsewhere ${ }^{2}$ and so in this paper we focus on how we have realised the design in hardware and software. We also present our results from recent performance tests in the laboratory and review the impact of these test results on the overall capability of the MROI. In section 2 we remind the reader of the most important functional and technical specifications for the FTT/NAS, and we then lead onto a description of the as-built system in section 3 . The results of our testing are presented in section 4 and our conclusions are given in section 5 . 


\section{FUNCTIONAL AND TECHNICAL REQUIREMENTS}

The MROI FTT/NAS has three functions:

- To sense the atmospheric- and mechanically-induced tip-tilt fluctuations at each UT, so as to provide control inputs to the UT active secondary mirror and thereby reduce these fluctuations;

- To act as a high sensitivity acquisition camera for each UT;

- To act as an accurate and precise beam angle sensor during the anticipated daily pre-observing co-alignment of the optical train of the MROI.

While the first of these roles is common to most FTT systems deployed in interferometric arrays, the second and third are direct consequences of the placement of the MROI FTT/NAS at each of the UTs. Usually, tip-tilt sensors in optical/IR interferometers interrogate the light from each UT after it has propagated to the beam combining laboratory. This allows for sensing of angle-of-arrival fluctuations arising from both the atmosphere and any relay optics between the UTs and the beam combiner. At the MROI, we have adopted a different approach by placing the FTT/NAS before the beam relay optics, in a thermally-unstable environment. This places challenging constraints on the stability of the beam relay system but allows for a single camera to fulfil the three roles outlined above, thereby saving costs. A second unconventional design feature of the FTT/NAS is the use of an opto-mechanical arrangement with no active elements. By excluding the possibility of adjusting any of the FTT/NAS optical components during the night, we have reduced complexity and costs, but this has required very careful design. Other aspects of the MROI FTT/NAS are less unusual. For example, it uses dichroic splitting to pick off the light for sensing, it exploits an EMCCD detector head to maximize sensitivity, and it controls a small tip-tilt mirror located essentially at an image of each UT pupil.

In terms of the derived technical requirements for the FTT/NAS, two have proved most challenging to deliver. These are (a) the need to maintain a stable guiding center on the tip-tilt sensor in the presence of the temperature changes expected inside each UT enclosure, and (b) the desire to realise a very low level of residual tip-tilt error for faint targets so as to maintain good sensitivity. More precisely these two criteria imply:

1. That the optics, dichroic and the sensor camera are sufficiently stable in tilt and displacement that in presence of a temperature change of $5{ }^{\circ} \mathrm{C}$, the guiding center will move by no more than 0.015 seconds of arc (i.e. roughly half a micron on the detector surface);

2. That for a point source target with a visual magnitude of 16 , and colours typical of a Type 1 Seyfert galaxy (i.e. with a spectral index $\alpha=-0.5$, where $F_{\lambda} \propto \lambda^{\alpha}$ ), the residual two-axis tip-tilt error after correction by the FTT/NAS will be no more than 0.060 seconds of arc. This assumes a value of $14 \mathrm{~cm}$ for $r_{0}$ at $500 \mathrm{~nm}$ and a wind speed of $10 \mathrm{~m} / \mathrm{s}$.

A considerable amount of what follows reports on design adjustments required by, and testing needed to validate, these two highest level performance goals. Fortunately, our preliminary testing does suggest that the system we have designed will come very close to meeting these requirements while simultaneously meeting the many others that we have not had space to mention here.

\section{DESIGN}

The basic architecture of the FTT/NAS has already been described before ${ }^{2}$ and so in each sub-section below we have simply summarised the most important aspects of the final implementation. In some places certain details have been omitted, but we have tried to describe those features of our implementation that will be of most interest to other interferometric sub-system designers. 

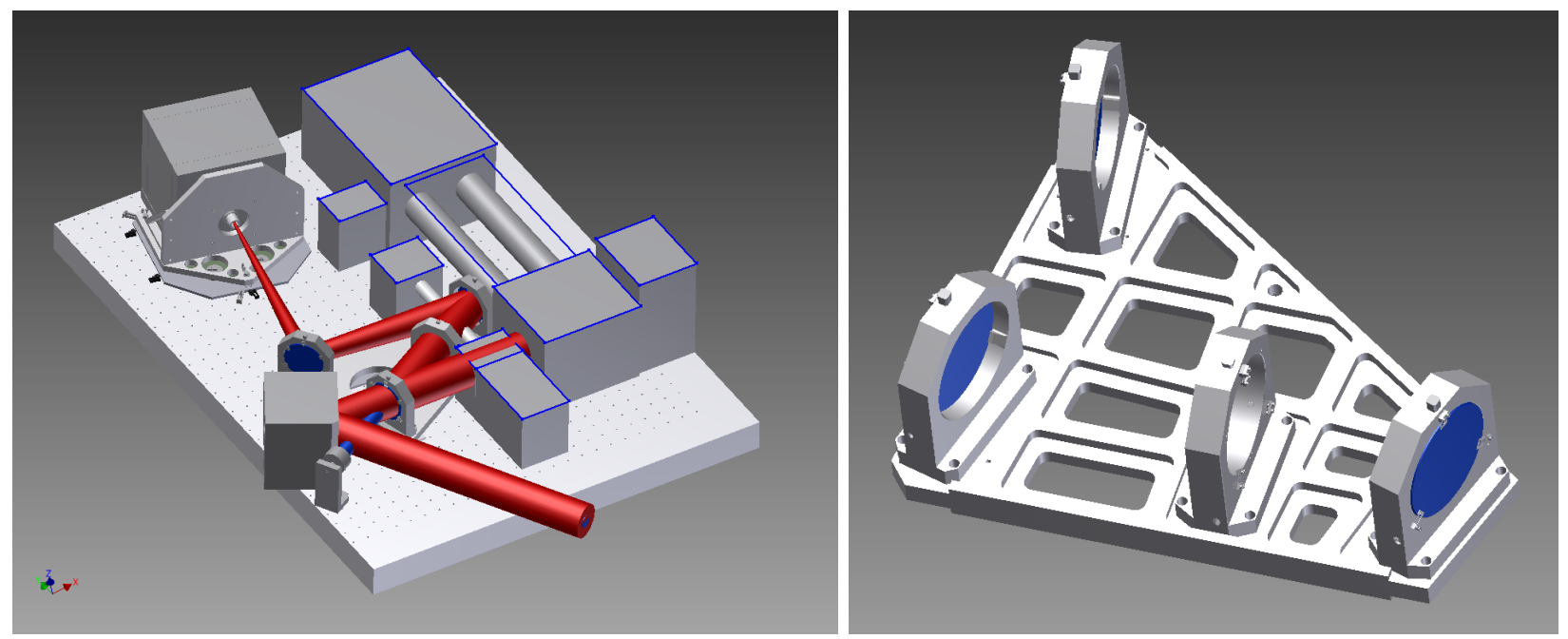

Figure 1. The location and geometry of the FTT/NAS on the UT optical Nasmyth table (left panel). Unrelated components on the table are shown as featureless boxes. The beam from the UT tertiary mirror enters via the boxes at centre-right and is intercepted by the dichroic to the right of the M4 mirror (cubic box at bottom left corner of table). The right hand panel shows the light-weighted optical base-plate together with the mounts for the dichroic, apochromatic lens, and the two fold mirrors. In this panel, the $95 \mathrm{~mm}$ diameter UT beam enters from lower center, propagating to the left. It then is reflected off the dichroic towards the right, passes through the lens, is reflected towards the left again, before being diverted to the camera head by the fold mirror at top left.

\subsection{Optical design and layout}

Each FTT/NAS comprises two unconnected assemblies located on the Nasmyth table of the UT (see Figure 1). The first is a stiff base-plate on which all the optics are mounted, and the second is another base-plate which supports an EMCCD camera housed within a specially designed environmental chamber. The first base-plate supports a large custom dichroic, used to transmit wavelengths longer than $1 \mu \mathrm{m}$ to the beam combining laboratory, together with the focusing lens and two fold mirrors that steer the shorter wavelength light to the camera head. The strict stability requirement on the guiding center means that the telescope beam, dichroic, lens, fold mirrors and camera have to maintain a very precise relationship to each other during the night. The design of these two assemblies has been optimised such that the geometry of the whole optical layout is invariant to temperature changes at the level specified in the top-level FTT/NAS requirements. The design features that achieve this invariance to temperature change are described in the following sections.

\subsection{Opto-mechanical design}

To minimise the effect of localised tilts of the Nasmyth optical table the mounts for the FTT/NAS optics are supported on a stiff base-plate (see Figure 1). All these components made from high thermal conductivity aluminium alloy, so as to minimise any spatial temperature differentials as the ambient temperature changes. The dichroic and fold mirror mounts share a number of features to further reduce their sensitivity to spatial temperature variations. First, in both cases the optics are sprung loaded onto three small polished seats located about the mount's internal circumference so that the reflecting surface lies in a vertical plane passing through the centre of the mount. Second, the optics are supported radially at two locations using materially compensated fingers that ensure that the optics remain centered in their mounts, minimizing any differential motion between the reflective faces and the seats.

The UT beam is focused by a custom $1.53 \mathrm{~m}$ focal length cemented triplet. This uses three common and easily worked glasses (N-BAK4, N-KZFS4, and N-LAF2) to give excellent achromatic performance from $400 \mathrm{~nm}$ to $900 \mathrm{~nm}$ and a temperature-dependent focal length that largely compensates for the thermal expansion/contraction of the steel top of the Nasmyth optical table. The lens mount also incorporates radial material 
compensation, to maintain centration of the lens despite the difference in CTE between the glass of the central lens element and the aluminium mount, and spring loading in the longitudinal direction.

The implementation of the base-plate had to balance stiffness and isothermal performance. In the presence of changes in temperature that might be experienced at the MROI site, static friction between the kinematic interfaces attached to the base-plate and the (steel) optical table led to vertical bending of a solid $25 \mathrm{~mm}$ thick aluminium base-plate, and hence differential tilting between the optical elements, a factor of 20 greater than allowed. As a result, the final base-plate is a light-weighted piece, milled from a single piece of stress-relieved aluminium selected for high thermal conductivity. It incorporates stiffening ribs, but has vertical symmetry about its mid-plane so as to confine any residual kinematic forces to that plane.

To reduce the magnitude of the frictional forces between the base-plate and the optical table top, the prototype kinematic interfaces were also modified so as to include rolling elements. The interfaces are based on the conventional ball, cone and "vee" principle, but utilise hardened steel components and balls so as to reduce "stiction". More specifically:

- The cone, embedded in the base-plate, together with its hardened steel ball, acts as a pivot located precisely under the reflecting surface of the last fold mirror and in the mid-plane of the base-plate. This fixes the relationship of the base-plate with respect to the camera mount as the optical table expands/contracts with temperature.

- The ball and vee seat comprises two co-aligned vees, one inverted and set into the base-plate and the other fixed to the optical table, with a steel ball that is free to roll placed between them. The vees are aligned so that their extended center-lines pass through the centre of the cone seat. As a result, if the base-plate expands/contracts relative to the table top, it does not rotate about the cone seat.

- The final seat uses two hardened steel flats, one fixed to the optical table and the other to the base-plate, with a hardened steel ball between them. This allows low-friction motion in any direction in the mid-plane of the base-plate.

- All the base-plate seats are accurately set at its mid-plane.

The second assembly on the Nasmyth optical table is the camera mount (see Figure 2). This is constructed of the same grade of aluminium as the base-plate so as to precisely match its response to temperature changes. The EMCCD head is held within a thermal enclosure on a sub-plate supported by pillars of low thermal conductivity composite that pass through the insulation to the camera mount. Similarly, a tube fixed to the sub-plate also passes through the insulation and on through a hole in the camera mount to allow the focused UT beam to reach the camera. A window mounted within this tube isolates the air within the camera enclosure from the ambient environment. Compliant seals surround the tube and sub-plate support pillars, ensuring that any movement of the enclosure is decoupled from the camera and its mount.

The camera mount itself is connected to the Nasmyth optical table via a steel interface plate. This uses a three ball and "vee" kinematic seat arrangement which maintains the position and orientation of the detector plane with respect to the common base-plate and ensures stability of the camera in the horizontal plane. The steel interface plate has the same CTE as the optical table and can be simply clamped in the required position relative to the base-plate and at the correct focal distance. To ensure that the camera also tracks the vertical displacement of the lens on the common base-plate the kinematic seats and interface plate use equivalent material vertical path lengths to that of the common optical base-plate.

To allow the EMCCD head to operate within the UT enclosure at temperatures below $0{ }^{\circ} \mathrm{C}$ it is housed in a thermally insulating, air-tight enclosure. The internal temperature of the enclosure is controlled via a chilled fluid loop provided by the UT. This not only prevents the camera from overheating, but more importantly limits thermal dissipation from the camera into the telescope enclosure.

The camera housing is made from rigid polyisocyanurate thermal insulation panels with a hard protective skin of fibre-glass board glued to its outer surface. It is supported above the optical table by a sheet of low 

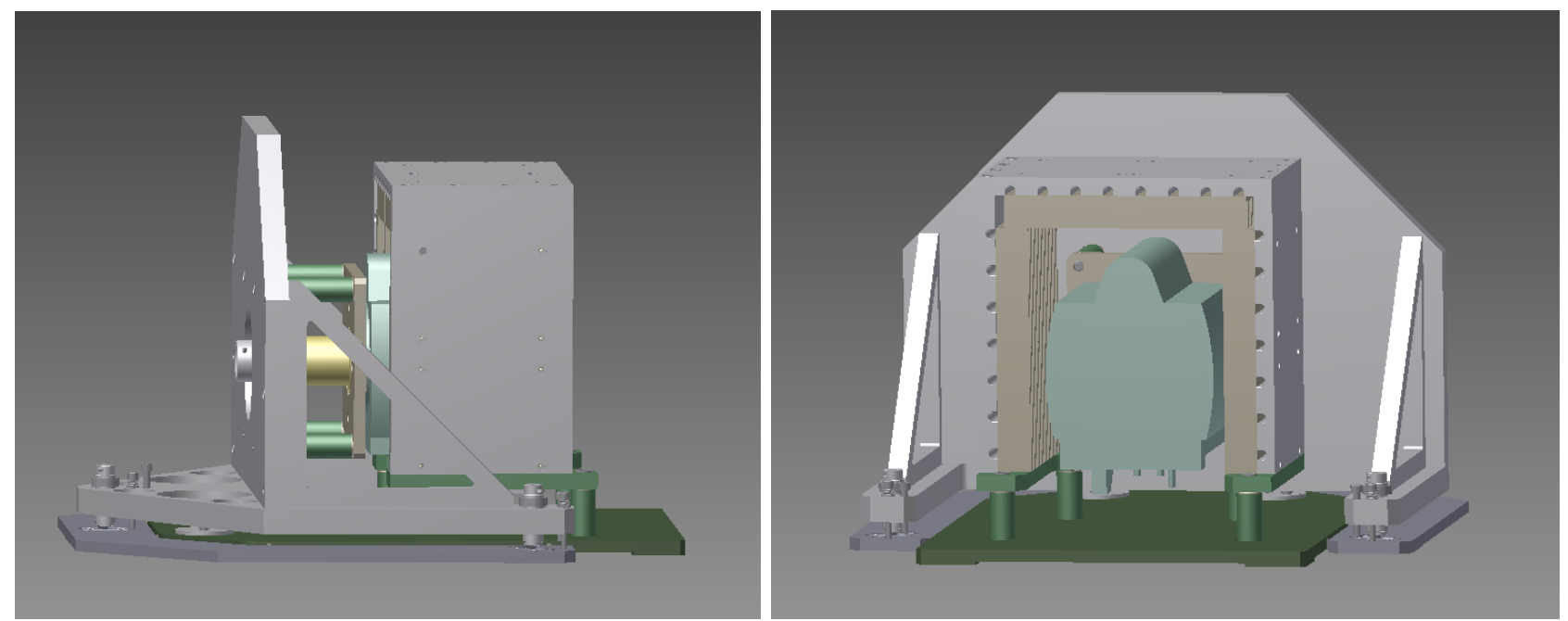

Figure 2. Side (left panel) and rear (right panel) views of the aluminium camera mount and the cold plate assembly with the enclosure removed. The four insulating pillars connecting the camera to the large camera sub-plate are visible in the side view, extending horizontally and surrounding the tube within which the light reaches the EMCCD. The similar vertical insulating pillars, seen in the rear view, support the cold plate assembly - used to regulate the camera enclosure temperature - which sits on an insulating base plate located within the steel interface plate of the camera mount.

thermal conductivity G10 fibre-glass. A custom designed heat exchanger sits within the enclosure to remove heat from the air by natural convection. This comprises an assembly of cold plates which surround the camera on three sides. The cold-plate assembly is mounted on a frame supported by four low thermal conductivity pillars which pass through the insulation and are fixed to the G10 plate below.

A number of other features are incorporated in the camera enclosure to provide environmental and protective control that is monitored and controlled by the FTT/NAS computer. These include (a) a dry air supply to flush the enclosure and (b) a resistive heating element that can be used to heat the enclosure in the event that it is too cold to start up the camera (see Figure 3 for further details).

\subsection{Software design}

The design of the FTT/NAS software is described in detail in our previous paper, ${ }^{2}$ and only a brief overview is given here. The system software runs on a rack mounted Intel-style computer housed in the UT enclosure. The operating system is expected to be Linux 2.6.32 with Xenomai 2.6 kernel patches, but the source code is compatible with more recent releases. Besides interfaces to the fast steering mirror and environment sensors, the computer has a Gigabit Ethernet connection to the MROI control room for communication with the Interferometer Supervisory System (ISS) and operator GUIs.

The software comprises two components used to control the FTT/NAS hardware, a user interface software application, and a fourth component used for offline visualization and analysis of previously-recorded monitor data. The control software is partitioned into two components because of the need to maintain the environmental conditions within the FTT/NAS camera enclosure at all times. Hereafter we will refer to the part controlling the camera environment and enabling/disabling the camera as the "environment controller", and the component performing the primary FTT/NAS functions such as target acquisition and fast tip-tilt correction as the "system controller".

The environment controller is a simple sensing application which reads and reacts to data on timescales of the order of one second, and hence runs in ordinary Linux. The application communicates with sensors (either analogue voltage or $\mathrm{I}^{2} \mathrm{C}$ types) and a heater via two USB-driven Labjack U3 boards.

The control/display GUI provides a graphical user interface for commanding the system and environment controllers and for live display of their monitor data (including camera images). It can also record monitor data 


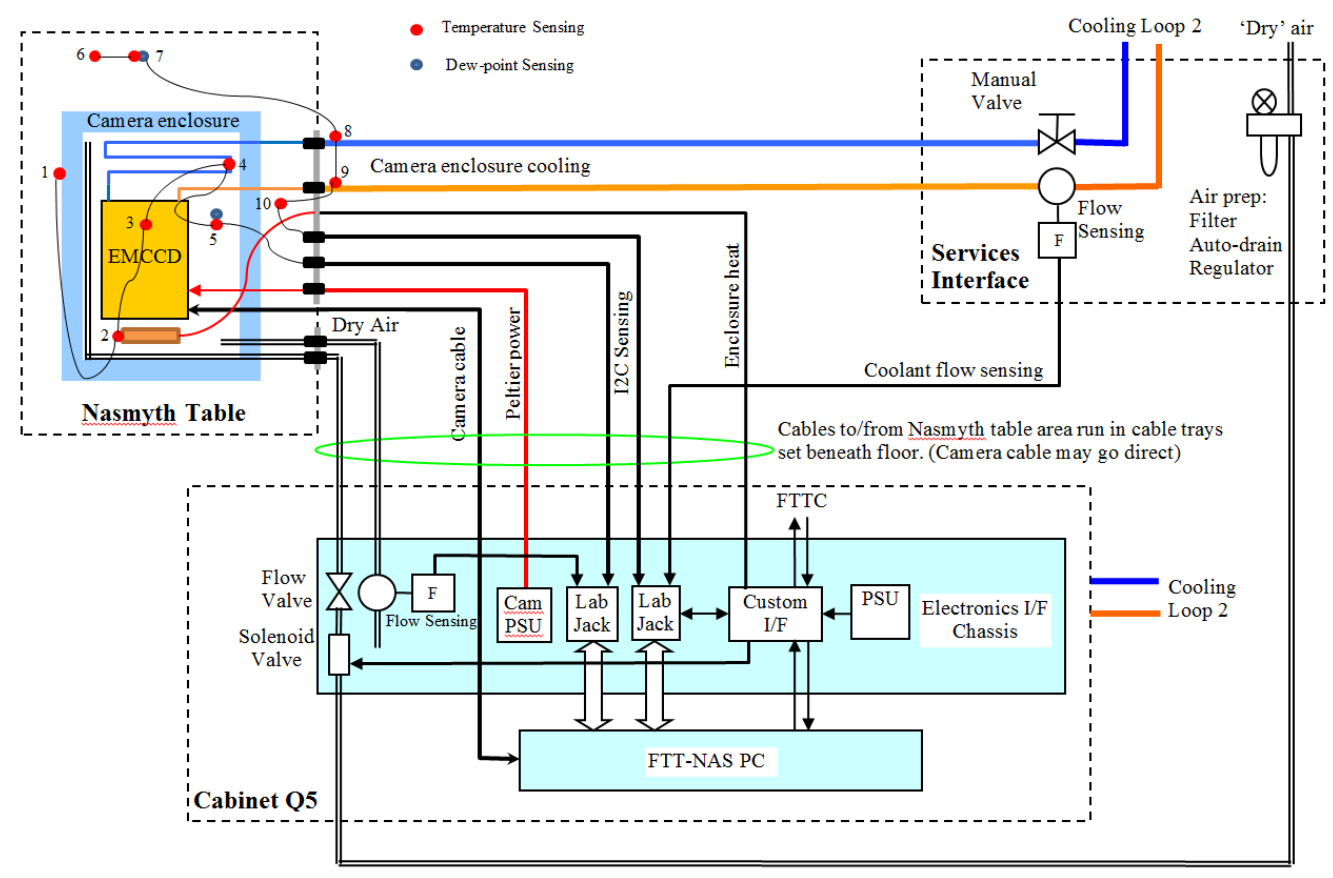

Figure 3. A schematic of the connections between the FTT camera enclosure, the air and coolant services and the electronics located in the temperature controlled telescope enclosure cabinet. Temperature and humidity sensors, connected via an $\mathrm{I}^{2} \mathrm{C}$ bus, are used to automatically shut down, or prevent start-up of the camera, if conditions are unsuitable. Note that the FTT/NAS interface electronics and its CPU sit in a the temperature controlled rack - cabinet "Q5" - within the telescope enclosure. A separate services interface plate (top right) manages the dry air and cooling water for the system and includes manual valves and a flow sensor.

to a set of FITS-format files when requested to do so by the operator. The control/display GUI may be used in one of two modes: a fully-functional "standalone" mode, and a "display-only" mode which can safely be used when the FTT/NA system is under the control of the central ISS.

The analysis GUI is used to visualize and process monitor data previously recorded to FITS files by the control/display GUI. The application runs under the Matlab environment and provides flexible capabilities for displaying time series of scalar data and images.

At the time of writing (June 2014), implementation of the system controller, environment controller and control/display GUI is mostly complete and testing is in progress. The analysis GUI has been partially implemented.

\subsubsection{System controller implementation}

The system controller software performs acquisition functions, fast tip-tilt servo loop closure and transmission of system diagnostic information (including camera images) over the network. This software can be broadly divided into two parts:

- Code that implements the real time fast tip-tilt servo loop using Xenomai. This is implemented as one kernel space thread and one user space thread.

- Code that implements supporting functionality, including network control and monitoring, using several non-real-time threads in Linux.

The core function of the software is closure of the servo loop between the EMCCD camera and the fast tip-tilt mirror. This software reads a camera image, calculates a centroid and then a correction, and sends the correction to the controller for the mirror. All this occurs in real time context. An interface is also provided to the remainder of the fast tip-tilt software, which runs in Linux. The implementation is illustrated schematically in Figure 4. 


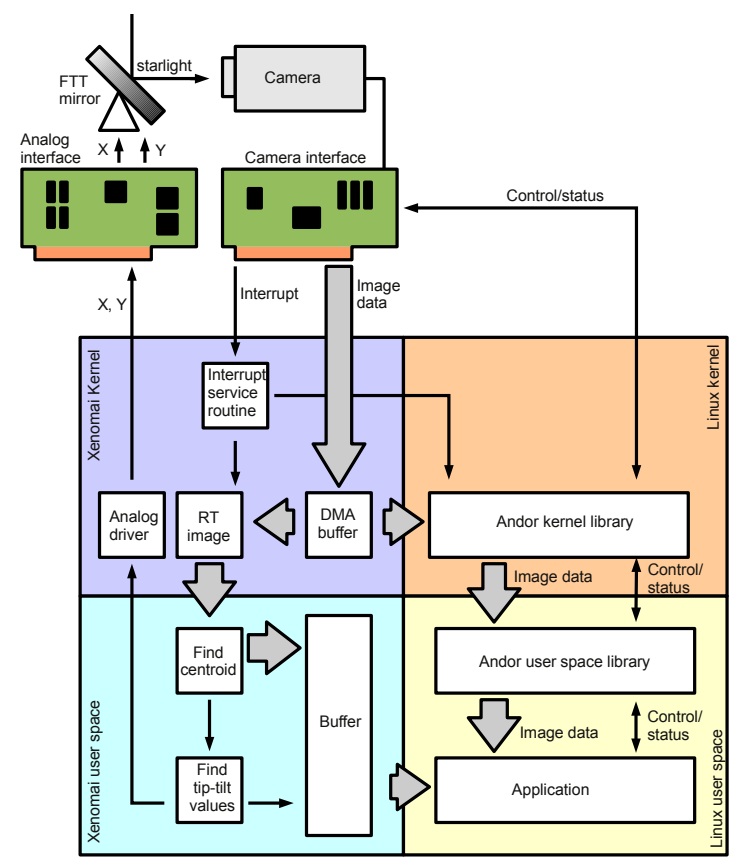

Figure 4. The implementation of the core servo loop in Xenomai and its connection with non-real-time Linux code.

The readout of the EMCCD is performed using custom firmware that collects date from a user-selectable 32 $\times 32$ Region Of Interest (ROI). This custom scheme allows for frame rates of up to $1140 \mathrm{~Hz}$, almost three times faster than that available with the default clocking scheme. This means that a $50 \mathrm{~Hz}$ closed-loop bandwidth can be realised while still using using a sufficiently large subframe to accommodate image motion (due to field rotation) of an off-axis guide target during a 300 second observation. The custom clocking scheme minimises the number of serial clock shifts required to get the data out of the ROI, at a cost of summing charge from pixels outside the ROI with charges from earlier rows of the ROI that have previously been clocked into the serial register. Normally there will not be a celestial object outside the ROI, but the summing of charge from multiple pixels will increase the level of spurious signals such as thermally-induced or clock-induced charge. Lab testing of the camera readout revealed a non-uniform background signal, which we have successfully modelled assuming a constant contribution from each CCD pixel that is summed into the output.

When the camera interface card reads out a camera image, it writes the data to computer memory using direct memory access and then triggers an interrupt to notify the computer that the transfer is complete. This interrupt is intercepted by an interrupt service routine which then allows real time access to image data. The interrupt service routine copies the image data to a buffer and blocks a Xenomai user-space routine from reading the data until the copy is complete. When the user-space routine is allowed to read the data, it does so immediately, firstly reads the computer's NTP stabilized system clock to timestamp the data*, then calculating the image centroid, exploiting Xenomai's ability to use the CPU's floating point hardware in real time context in user space.

The offset due to the current tip-tilt mirror position is subtracted from the image centroid, and the result is used as the input to a Kalman adaptive servo filter. The output of the filter is the best estimate of the current atmospheric tip-tilt. This is converted into a demand position for the UT secondary mirror and sent to a custom Xenomai driver for the analogue interface card, which applies scaled voltages to the FTTA controller inputs. Meanwhile the image data and corrections are buffered into an ordinary Linux application, where they are used for non-real-time tasks and logging. This buffering serves as an interface between the Xenomai code, which must meet strict deadlines, and the Linux code, which only needs to keep up "on average".

\footnotetext{
* Xenomai V2.6 or later is needed to read the system clock in real time context and hence provide an accurate timestamp.
} 


\section{PERFORMANCE TESTING}

Our approach to testing the FTT/NAS has involved both individual component tests and where possible subsystem tests where combinations of components are tested for functionality and performance. The most important of these are summarised below.

\subsection{Camera sensitivity testing}

The sensitivity goal for the FTT/NAS derives from an error budget comprising a number of terms each contributing to an overall two-axis tip-tilt residual error that must not exceed 0.06 seconds of arc for a target with a $V$ magnitude of 15.8. This budget is shown in Table 1. Here, the "detection noise centroiding error" term includes the effects of photon shot noise and (negligible) detector readout noise and assumes a target with typical Type 1 AGN colours $\left(F_{\lambda} \propto \lambda^{-0.5}\right)$. The optical throughput is $65 \%$ from the top of the atmosphere to the detector (the throughput of the FTT system optics is 86\%) and a bandpass from 600-1000 nm has been assumed.

\begin{tabular}{lr} 
Tilt error & RMS error/milliarcsec \\
\hline UT residual mount error & 20 \\
UT residual wind shake & 30 \\
Residual seeing tilt & 30 \\
Speckle noise centroiding error & 15 \\
Detection noise centroiding error & 34 \\
\hline Total & 60
\end{tabular}

Table 1. The original MROI two-axis tip-tilt error budget for the FTT/NAS in seeing conditions of $r_{0}=14 \mathrm{~cm}$ and $V=10 \mathrm{~m} / \mathrm{s}$. In the course of the detailed design phase of the FTT/NAS we have established that a more realistic estimate for the contribution to the speckle noise centroiding error at low light levels is approximately 40 milliarcsecond. This would increase the total budget from 60 to 70 milliarcseconds. For the purposes of this document, however, we will continue to assume a total two-axis budget of 60 milliarcseconds.

We have assessed the expected performance of the FTT/NAS in a number of different ways. First, we have undertaken a closed-loop computational simulation of the system to see how a "perfectly" performing EMCCD would behave when operating at the photon rates appropriate to our desired limiting sensitivity. Second, we have tested the open loop centroiding performance of the actual EMCCD head when operating at high frame rate and low light levels. Finally, we expect to compare the measured closed-loop performance (see Sec. 4.5) with the error budget predictions in the very near future.

Our computational simulation incorporates a Kolmogorov phase screen translated at a constant velocity past a UT aperture. This was used to generate simulated CCD images of a point source guide target with realistic speckle aberrations. Pseudo-random noise was added to the synthetic images, which were then processed using algorithms that are identical to those that have been coded for the real-time system controller. The simulation included realistic levels of readout and actuation delay, detector readout noise, and the spatially modulated clockinduced charge (CIC) background associated with the custom EMCCD readout. However, it ignored quasi-static aberrations in the UT, the effects of atmospheric dispersion, and any additional random non-uniformities in the clock-induced charge background.

Our current simulation results show that the residual non-UT-related contributions to the two-axis tip-tilt error budget can be met, but only down to target magnitudes of $m_{V} \sim 14$. At a magnitude of $m_{V}=15.8$ we predict tip-tilt residuals roughly a factor of 1.8 times greater than anticipated. We believe this is due to two factors. Firstly, a larger than anticipated contribution from the speckle noise centroiding error arising from crosstalk between the coma and tilt components of the atmospheric fluctuations ${ }^{\dagger}$. Secondly, an additional

\footnotetext{
${ }^{\dagger}$ When preparing the error budget we had assumed the possibility of eliminating some of the crosstalk between the coma and tilt components of the atmosphere through a judicious choice of "centroid" algorithm (for example windowing or thresholding to exclude the outer regions of the stellar image). Our simulations suggest that while this is possible at higher light levels, these methods deliver little or no gain at the sensitivity limit.
} 


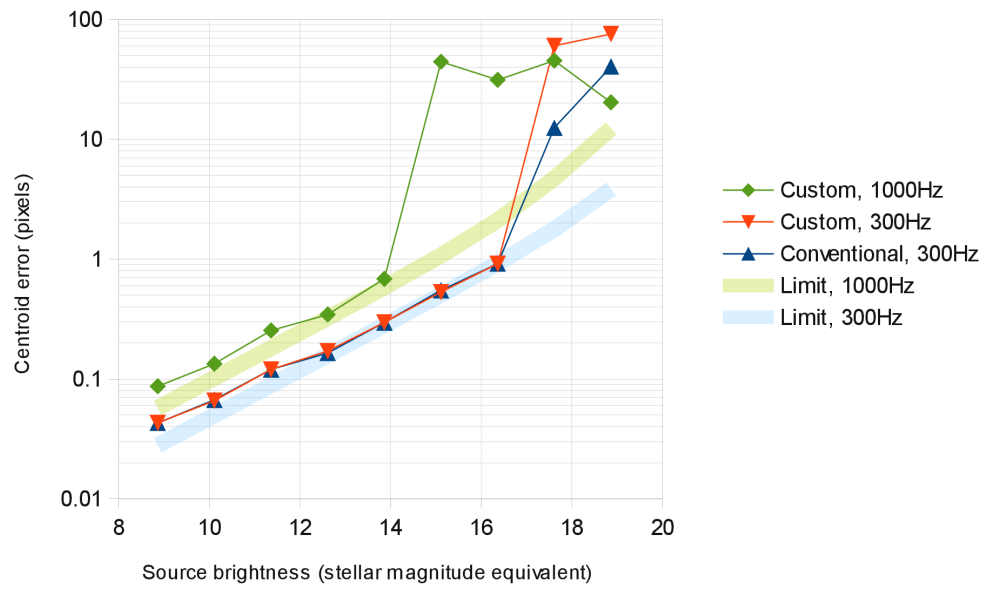

Figure 5. Two-axis centroid error as a function of (a) the EMCCD clocking scheme; (b) the source magnitude at the MROI and (c) the frame rate. The theoretical limits to centroiding performance for frame rates of $300 \mathrm{~Hz}$ and $1000 \mathrm{~Hz}$ are also shown. Due to the uncertain flux calibration of our experimental set-up these predictions are only accurate to the $10 \%$ level, and hence these limits are shown as shaded bands. Note that at a $300 \mathrm{~Hz}$ frame rate, the EMCCD essentially behaves perfectly for targets as faint as $m_{V}=16$.

contribution to the detection noise centroiding error due to the stochastic amplification of the CIC background, which again had not been anticipated when the original tip-tilt error budget was prepared. We have simulated the latter effect using parameters from the EMCCD datasheet, which still need to be checked against the measured performance of the detector.

A parallel check on the EMCCD centroiding performance was undertaken by measuring the variation in openloop centroid jitter for a stationary diffraction-limited image as a function of light level (see Figure 5). For the custom clocking mode at a $300 \mathrm{~Hz}$ frame rate, the measured jitter was consistent with theoretical expectations for the effects of photon shot noise and readout noise on an EMCCD (and excluding CIC), down to a light level equivalent to a magnitude 16 target. At a $1 \mathrm{kHz}$ frame rate this threshold light level rose to approximately magnitude 14. Results obtained using both our custom ROI readout mode and conventional ROI readout modes gave indistinguishable results at frame rates of $300 \mathrm{~Hz}$, suggesting that the use of our custom readout scheme should not have any substantive impact on the limiting sensitivity of the system.

\subsection{Opto-mechanical stability testing}

In our previous paper ${ }^{2}$ we reported testing of the mechanical stability of the individual FTT/NAS optical elements in their mounts. These preliminary tests established that the focusing lens and mount met all their stability requirements but could only demonstrate that the dichroic and fold mirrors were stable at a level no worse than twice that required. In the following paragraphs we summarise the results from "integrated" tests in which the dichroic, lens, and two fold mirrors were tested as a complete unit, i.e. mounted on their light-weighted aluminium base-plate.

\subsubsection{Integrated test arrangement}

Integrated testing of the common base-plate assembly was executed by placing the whole unit in an environmental chamber, and feeding it with collimated light from a test source located on a separate optical table. The dichroic and two fold mirrors were replaced with otherwise identical semi-silvered substrates which allowed the beam to be interrogated after each reflection (see Fig. 6) and at the location of the EMCCD camera head. 


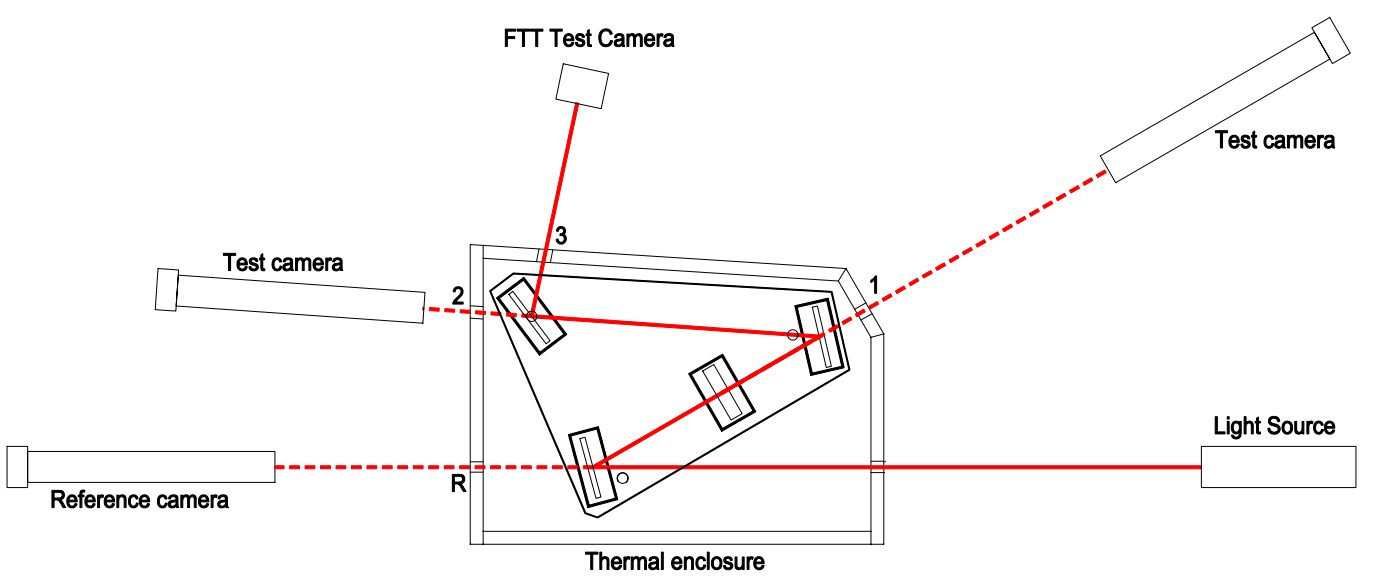

Figure 6. A sketch of the extended integrated test arrangement. The collimated light source is mounted on a separate optical table and the dichroic and fold mirrors "leak" some of the test beam to cameras placed behind them. These three test cameras monitor the reference beam at "R", after the first reflection at "1", after the second reflection at " 2 " and after the final reflection off the second fold mirror at "3". Tests can be run either without the FTT/NAS lens - in which case the cameras are fitted with $500 \mathrm{~mm}$ lenses — or with it, in which case only the camera at location $\mathrm{R}$ requires a lens.

The chamber was cooled by circulating air through it originating from a separate heat exchanger. However, it proved almost impossible to achieve isothermal cooling of the chamber despite considerable effort and so for all of our tests we monitored the temperature of either side of the top of the base-plate so as to check for any temperature differences that might lead to warping of the base-plate in the plane of the plate. A typical test involved a cooling cycle starting with the chamber at just above room temperature and then forcing it to cool by about $5{ }^{\circ} \mathrm{C}$ over roughly 12 hours. The chamber was then allowed to recover passively to room temperature. In such a cycle the initial cooling rate was always much greater than the $1.5^{\circ} \mathrm{C} /$ hour the FTT/NAS is specified for, but the initial recovery typically matched those conditions.

The geometric configuration of the dichroic and first fold mirror, in an almost anti-parallel arrangement, meant that any tilting of the optics whether due to their mounts or the base-plate bending was immediately detectable by subtracting the position of the reference beam at $\mathrm{R}$ from the beam delivered to port 2. Similarly, by combining the beam positions measured at all the port locations, and swapping the locations of the dichroic and fold mirrors, we were able to confirm the stability of all of the optics including the FTT/NAS lens.

This second process was less than straightforward, primarily because additional calibrations had to be made to accommodate the fact that (a) the cameras interrogating the beams were not inside the environmental chamber and (b) that the forced cooling of the environmental chamber led to distortion of the optical table on which the experiment was set up. Furthermore, different realisations of the tests indicated that the level of residual opto-mechanical stability in the horizontal direction was directly related to the differential temperature measured across the common base-plate ${ }^{\ddagger}$. Repeat tests allowed us to establish the scaling between $\Delta T$ and any residual $x$-deviation, and so while we do not expect that $\Delta T$ will ever be as large on site as we have experienced in our tests, we are confident that its impact on the opto-mechanical stability of the FTT/NAS can be managed at the level needed to meet the system requirements.

\subsubsection{Integrated test results}

Fig. 8 shows calibrated measurements of the motion of the reference beam at the location of the FTT/NAS camera in the $x$ and $y$ directions. These were recorded over the course of a typical cooling/warming cycle of 16 hours duration. A typical calibration consisted of subtracting the reference beam deviations (suitably scaled and inverted where necessary due to co-ordinate inversions and differences in the focal lengths of the lenses) from the FTT/NAS sensor deviations, applying a correction for the change in height of the centre of the lens mount due

\footnotetext{
${ }^{\ddagger}$ This correlation arises because the spatial temperature gradient causes the common base-plate to distort in its own plane, thereby leading to very small changes in the azimuths of the dichroic and fold mirror mounts.
} 


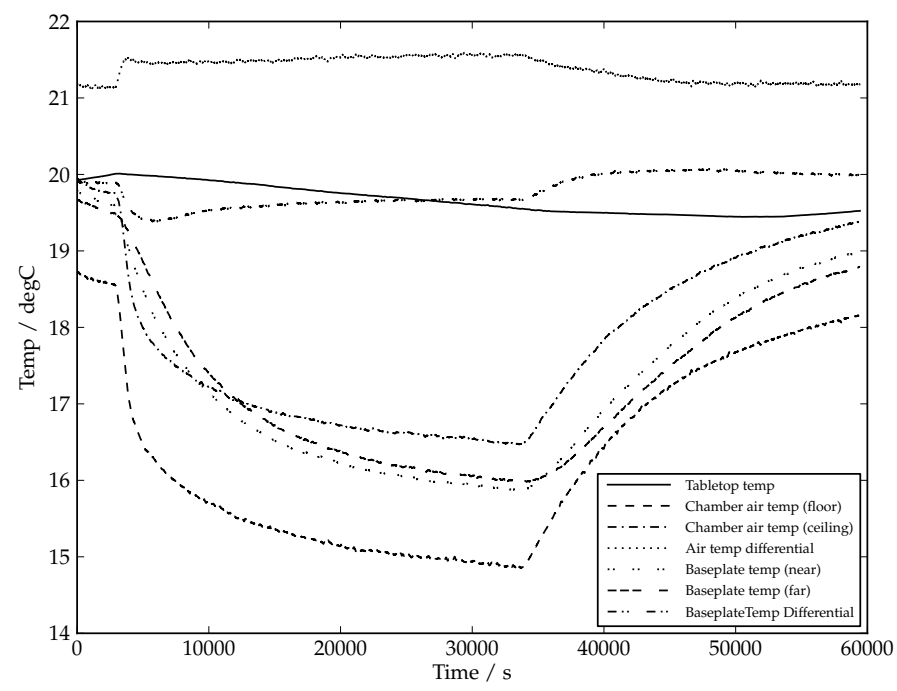

Figure 7. Test chamber and base-plate temperatures obtained during a typical test cooling cycle. Note the high rate of change of temperature at the start of the cooling and the slower rate of change of temperature when warming occurs. In this example, a small $\left(\Delta T \sim 0.4^{\circ} \mathrm{C}\right)$ but significant differential temperature appears across the base-plate when the forced cooling is initiated since the chilled air enters the chamber closer to one edge of base-plate than the others. This differential decays with time, but is only completely eliminated once the chamber has almost fully returned to its initial state.

to the temperature change within the environmental enclosure ${ }^{\S}$ and applying a correction for the depression of the optical table surface (these last two corrections are available directly from the test camera results).

In broad terms these results confirm that our design for the FTT/NAS is able to maintain the alignment of the key optical elements to a level that meets the MROI requirements. In the vertical $(y)$ direction, the typical uncontrolled excursions of the beam were within specification. In the horizontal $(x)$ direction the beam direction excursions exceeded the requirement by a factor of two, but in all of our tests we were never able to eliminate differential temperatures across the width of the base-plate and so this result is expected. Using a scaling obtained from several test results where the differential temperature varied between $0{ }^{\circ} \mathrm{C}$ and $1{ }^{\circ} \mathrm{C}$ we have determined that an empirical correction can be applied to reduce the measured $x$-deviation to $<0.1$ pixels. Hence a similar level of performance was realised in this coordinate, and so we are confident that on site the system will meet its opto-mechanical stability target.

\subsection{Software testing}

We have followed both top-down and bottom-up approaches to testing the functionality and performance of the FTT/NAS control software, in order to minimize the time needed for debugging on sky. The commands accepted by the system and environment controller applications were tested individually by suites of unit tests. Separate test suites have been written for the two communications interfaces (the ISS interface and the interface to the control/display GUI). For convenience, the unit tests can be run without hardware components such as the EMCCD camera, making use of emulations built into the applications.

We have tested the production implementations of the median centroid algorithm and Kalman filter by directly integrating the code into the simulator described in Sec. 4.1, replacing the original simulator implementations. We found that after debugging, the raw centroids and filter outputs were unaltered by this exchange of code.

\footnotetext{
$\S^{\S}$ At the telescope, the change in height of the lens mount with temperature is designed to exactly match that of the mount of the FTT/NAS camera. Since in our laboratory tests the camera emulating the FTT/NAS sensor sits outside the chilled enclosure, this "built-in" compensation needs to be backed-out.
} 


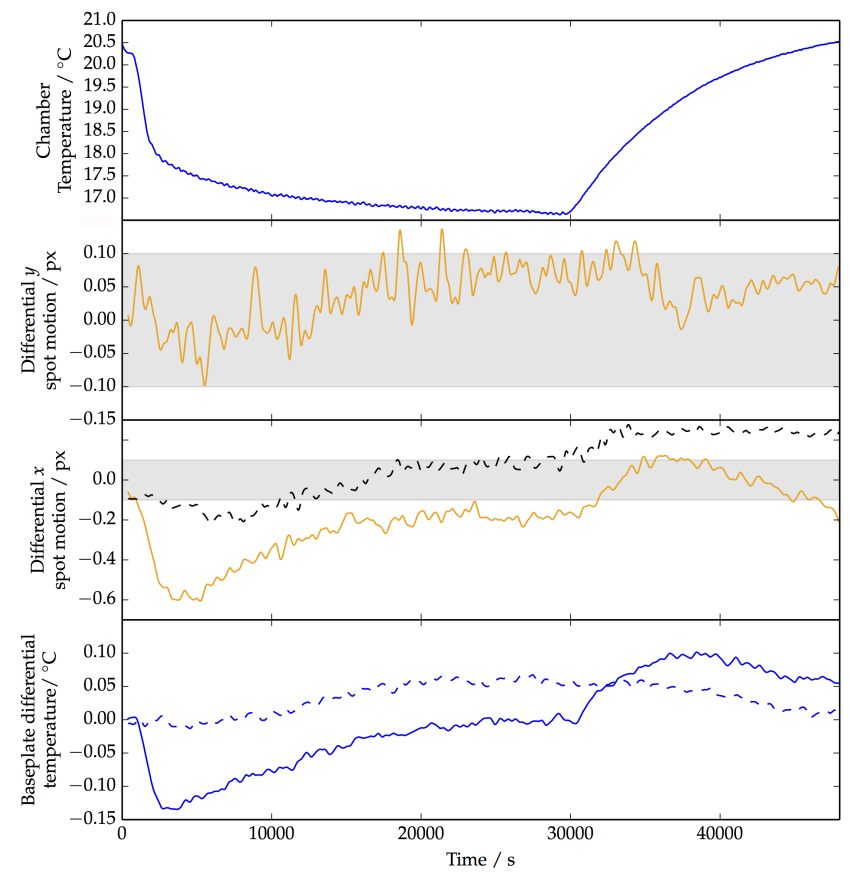

Figure 8. Calibrated results of image motion in the vertical $(y)$ direction (second panel from top) and horizontal ( $x$ ) direction (third panel from top) during some typical 16-hour thermal cycles. The shaded bands delineate the regions in which the stability requirement is met. The environmental chamber temperature is shown in the top panel and in these cases had a range of roughly $5{ }^{\circ} \mathrm{C}$. Two sets of data are plotted for the $x$ motion corresponding to different temperature differences measured between two sides of the common base-plate. The differential temperature profiles are shown in the bottom panel and reveal that the uncontrolled $x$ motions are very closely related to the spatial temperature variations. Our measurements suggest that the FTT/NAS opto-mechanical stability is within specification in the $y$ direction and is expected to meet specification in the $x$ direction as long as any differences in temperature across the common base-plate are small.

The system controller functionality has also been tested in aggregate, with real hardware, using the closed-loop test experimental setup described in Sec. 4.5.

Finally, we have also measured the delay introduced by the real-time software in responding to the interrupt generated by the Andor PCI card, and calculating and outputting a correction signal. For a dual core $3 \mathrm{GHz}$ AMD Athlon II computer, over many trials the maximum delay was measured to be $38 \mu \mathrm{s}$. We were able to run the custom readout at frame rates of up to $1140 \mathrm{~Hz}$, and so we expect to easily reach the goal for the system closed loop bandwidth of $50 \mathrm{~Hz}$.

\subsection{Camera enclosure thermal testing}

Our testing of the thermal enclosure for the EMCCD camera has focused on three main issues. First, that the thermal model we have for the enclosure is consistent with our laboratory measurements. Second, that the camera will be protected from temperatures outside its operating $\left(0^{\circ} \mathrm{C}\right.$ to $\left.30^{\circ} \mathrm{C}\right)$ and survival $\left(-25^{\circ} \mathrm{C}\right.$ to $55^{\circ} \mathrm{C}$ ) ranges. And, thirdly, that the predictions of our thermal model meet the requirements for the thermal performance of the camera enclosure for the full range of environmental conditions expected within the UT enclosure, in particular that, during operation, the external wall temperature of the camera enclosure remains within $2{ }^{\circ} \mathrm{C}$ of ambient over the range $-5{ }^{\circ} \mathrm{C}$ to $20^{\circ} \mathrm{C}$.

Our tests were conducted with a dummy heat source representing the camera so as to provide a controllable heat load. Tests on the camera itself confirmed that its heat dissipation depended on the readout rate and the residual dissipation from the camera, i.e. the heat not removed by the fluid cooling of the Peltier block. Typically, this was in the range $5-12 \mathrm{~W}$. All the runs took place at an ambient laboratory temperature of around $22{ }^{\circ} \mathrm{C}$ 
and used a recirculating chiller to provide cooling fluid approximately $3{ }^{\circ} \mathrm{C}$ below ambient. Temperature sensors monitored the camera mounting plate, the cold plate and the air at several locations within the thermal housing. The outer surface temperature of the housing was also monitored.

An example of a test used to validate our thermal model used $10 \mathrm{~W}$ of internal dissipation and no fluid cooling. At an ambient laboratory temperature of $23^{\circ} \mathrm{C}$ the internal air temperature of the housing reached $45^{\circ} \mathrm{C}$ after 8 hours of operation. After cooling was switched on the internal air temperature dropped to $24.5^{\circ} \mathrm{C}$ and the outer surface of the thermal enclosure quickly equilibrated to a temperature of $24^{\circ} \mathrm{C}$.

A more representative test, carried out at an ambient temperature of $23.5{ }^{\circ} \mathrm{C}$, involved first running the housing coolant loop for 1.5 hours and then increasing the internal heat dissipation from $0 \mathrm{~W}$ to $10 \mathrm{~W}$. In this case, the inner air temperature increased from $21^{\circ} \mathrm{C}$ to $25.5^{\circ} \mathrm{C}$ within an hour and a half, and the outer surfaces of the housing reached $24.5{ }^{\circ} \mathrm{C}$. For the next 16 hours the temperatures of the interior of the housing, the laboratory environment and the outer surface of the camera enclosure stabilized at $26{ }^{\circ} \mathrm{C}, 24.5{ }^{\circ} \mathrm{C}$ and $25{ }^{\circ} \mathrm{C}$ respectively, i.e. within spec. In this case, the coolant remained at $20.5^{\circ} \mathrm{C}$ and so the temperature differential between it and ambient had reached $4{ }^{\circ} \mathrm{C}$ by the time the experiment was stopped.

These, and similar tests, have established that our design for the EMCCD thermal enclosure does allow the camera to be maintained within its normal operating temperature range without its outer surface temperature rising more than $2{ }^{\circ} \mathrm{C}$ above ambient, even at ambient conditions as cold as $-5^{\circ} \mathrm{C}$. Finally, our modelling confirms that in operations on site the coolant temperature would not drop below $0^{\circ} \mathrm{C}$ whilst the camera was operating.

\subsection{Closed loop testing}

Preliminary tests of the fast tip-tilt loop have been carried out in the laboratory at Cambridge and are still ongoing as we work towards optimizing the system servo parameters. Our experiments have used an artificial target which has been perturbed in tip and tilt with representative Kolmorogov atmospheric fluctuations. The source has been imaged on the FTT/NAS EMCCD camera and stabilized in position using the FTT/NAS software to close a servo-loop with a fast actuated mirror.

Because the FTT opto-mechanical system (Sec. 3) is entirely passive - the fast steering mirror is the UT secondary - we have emulated the optical train of the UT \& FTT/NAS using a table-top optical train (see Fig. 10). This has used a Newport FSM300 fast actuated mirror (bandwidth $\sim 580 \mathrm{~Hz}$ ) to emulate the UT secondary which is mounted on a Physik Instrumente active hexapod. Both these devices accept an analogue voltage demand signal and both use position feedback.

Although we were able to correctly reproduce the image size and motion expected for an observation at the MROI in the lab setup, it does lack a few details that are expected to be present on sky. Most importantly, we used a monochromatic HeNe laser as the light source and did not attempt to introduce higher order aberrations into the beam or mimic the effects of atmospheric dispersion. We were able to calibrate the light level to $\sim 10 \%$ by measuring the signal with the EMCCD camera operating in photon-counting mode.

Typical results from an experiment run at a photon rate corresponding to an AGN-like target with a $V$ magnitude of 16 are shown in Fig. 10. In this case the measured single-axis jitter was measured to be at the 90 milliarcsecond level for a $300 \mathrm{~Hz}$ frame rate. This is a factor of roughly 2.5 above the requirement, but is consistent with our computational model for such an exposure time, which is perhaps a factor of three too short for use at the faintest light levels. Overall, these tests suggest that we should be confident of realising the jitter requirement for the FTT/NAS at a $V$ magnitude of $\sim 14$, but thereafter we expect to see a gradual degradation in performance, exceeding the design requirements for jitter by perhaps a factor of two at around magnitude 15.5 .

\subsection{Overall performance impact}

The results presented above show that the as-built FTT system is close to meeting its ambitious performance goals. The integrated stability test results show that the stability requirement is satisfied in the vertical direction, and will be met in the horizontal direction provided the temperature differential across the common base-plate can be maintained below $\sim 0.03{ }^{\circ} \mathrm{C}$. The impact of exceeding the stability requirement by a factor of two overall 


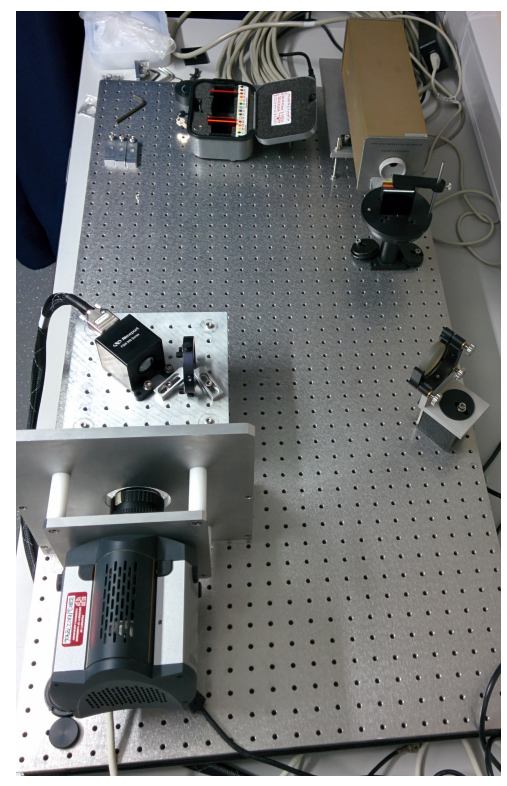

Figure 9. Photograph of the apparatus used for the closed-loop FTT/NAS experiment. The artificial target beam enters from the laser source at top right, is attenuated and then propagates as a collimated beam to the fold mirror at middle right, before being diverted horizontally to the active mirror at middle left. Just before the tip-tilt mirror, the beam is stopped down so as to provide the correct image size on the EMCCD. The focusing optic can just be seen attached to EMCCD camera head towards the bottom left of the picture.
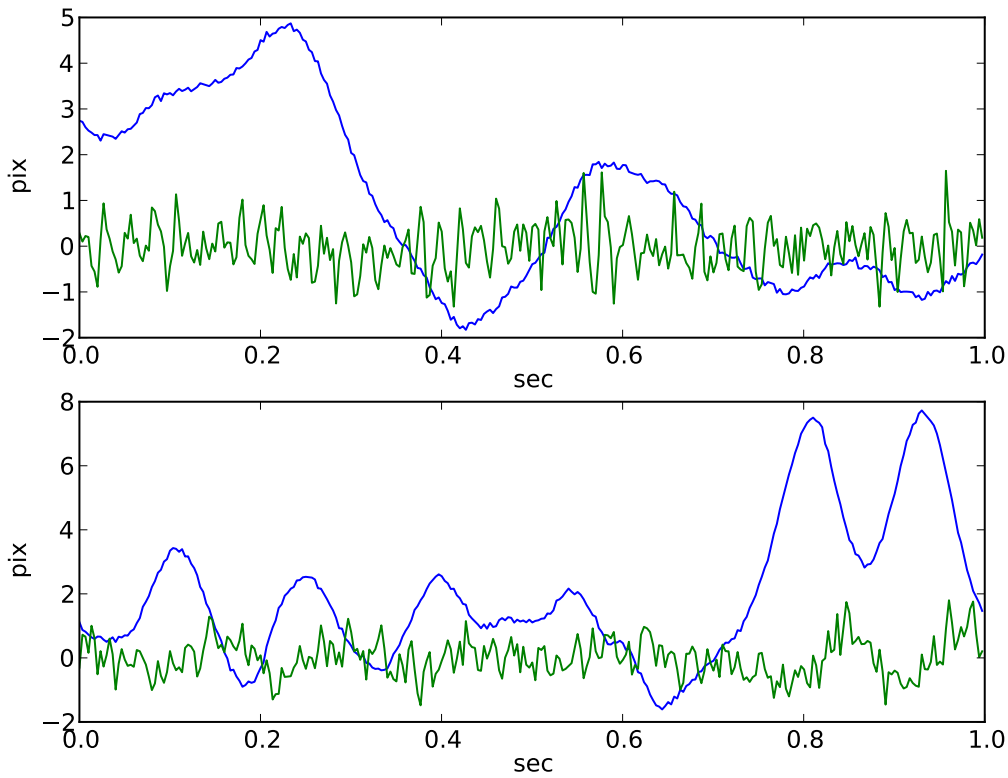

Figure 10. Tip and tilt disturbance signal (larger amplitude) and residual (smaller amplitude) measurements from the closed-loop experiment. For this test, tip and tilt perturbations for 0.65 arcsecond seeing and a wind speed of $10 \mathrm{~m} / \mathrm{s}$ were used, together with a light level appropriate to an AGN-like target with a $V$ magnitude of 16 . The $x$ and $y$ residual image jitters are very similar in magnitude. Their rms values are both approximately 0.6 pixels, i.e. equivalent to 0.09 seconds of arc on the sky. These preliminary results were secured using a frame rate of $300 \mathrm{~Hz}$, the standard EMCCD clocking mode, and a simple proportional servo. 
would be an additional $3.5 \%$ visibility loss (on top of the $1 \%$ originally budgeted). This could be eliminated by re-determining the tip-tilt zero point at intervals (of perhaps several hours) during the night, thus restoring the limiting sensitivity at the cost of observing efficiency. However the impact on the limiting sensitivity is so small that we would probably choose not to perform these additional calibrations.

The 60 milliarcsecond total two-axis error from the original tip-tilt error budget (Table 1) corresponds to a $15 \%$ visibility loss at $H$ band. If we only realize the current performance of the simulator, i.e. a 93 milliarcsecond two-axis error after adding in the UT-related terms, the visibility loss will instead be $36 \%$. This additional $21 \%$ visibility loss would result in a loss in $H$ band fringe tracking sensitivity of 0.4 mag. The tip-tilt error would be smaller if the level of clock-induced charge turns out to be lower than anticipated (there is some evidence for this from our measurements shown in Figure 5), or if we can develop a centroiding algorithm that is partially insensitive to coma at low light levels.

\section{CONCLUSIONS}

We have described the design of the MROI fast tip-tilt correction and target acquisition system. Locating the system at the telescope allows more photons to be collected, but exposes the opto-mechanics to a varying ambient temperature. This architecture has been validated by a comprehensive set of laboratory measurements, which show that the ambitious opto-mechanical stability requirements are met provided spatial temperature gradients are minimized.

The fast tip-tilt loop has been closed in the laboratory at light levels equivalent to a 16th magnitude guide target, albeit with larger tilt residuals than originally allowed for. We are continuing to optimize the closed-loop performance in preparation for on-sky commissioning and acceptance testing of the FTT/NAS in the fall of 2014.

\section{ACKNOWLEDGMENTS}

The Magdalena Ridge Observatory (MRO) interferometer is hosted by the New Mexico Institute of Mining and Technology (NMT) in Socorro, NM, USA, in collaboration with the University of Cambridge, UK. Previously funded by the Naval Research Laboratory (under Agreement No. N00173-01-2-C902), MROI is currently funded by the US Department of Transportation, the State of New Mexico and by New Mexico Tech.

\section{REFERENCES}

[1] Creech-Eakman, M. J., Romero, V. D., Payne, I., Haniff, C. A., Buscher, D. F., Alvidrez, V., Dahl, C., Deninger, J., Farris, A. R., Jimenez, S. R., Jurgenson, C. A., King, R., Klinglesmith, D. A., McCracken, T. M., Olivares, A. M., Salcido, C. D., Santoro, F. G., Seamons, J., Selina, R. J., Shtromberg, A. V., Steenson, J. M., Torres, N. C., Fisher, M., Seneta, E. B., Sun, X., Wilson, D. M. A., and Young, J. S., "The Magdalena Ridge Observatory interferometer: a status update," Proc. SPIE 8445, 84450P (2012).

[2] Young, J. S., Buscher, D. F., Fisher, M., Haniff, C. A., Rea, A. D., Seneta, E. B., Wilson, D. M. A., Farris, A. R., Olivares, A., and Selina, R. J., "The MROI fast tip-tilt correction and target acquisition system," Proc. SPIE 8445, 84451V (2012). 